\title{
ON THE SUPPORT OF HARMONIC MEASURE FOR SETS OF CANTOR TYPE
}

\author{
LENNART CARLESON
}

1. Introduction. In a recent paper [2] Kaufman and $\mathrm{Wu}$ have shown that the support of harmonic measure of the von Koch snowflake domain has dimension strictly smaller than the Hausdorff dimension $(\log 4 / \log 3)$ of the boundary curve. This result supports a very strong conjecture of Øksendal [4] to the effect that the support of any harmonic measure in the plane has dimension $\leqq 1$.

We shall here prove that the Øksendal conjecture is true for any "fractal" curve such as the snowflake. It is also true for any two-dimensional Cantor set with constant ratio in the very strong sense that the dimension for these supports is strictly $<1$ even when the Hausdorff dimension of the Cantor set is close to 2 .

If one thinks of harmonic measure as hitting probability it may not be so surprising that the results are consequences of results on stationary processes. More precisely, they belong to the field of information theory and we shall here review what is needed (see e.g. [1]).

After this manuscript was finished I was informed that A. Manning has a similar result for invariant measures of a polynomial map. (See [3].)

Let $x=\left\{x_{n}\right\}_{-\infty}^{\infty}$ be a double infinite sequence of symbols $x_{n}$ taken from a finite set, "alphabet". Let $\mu$ be a probability measure on the set of $x$ 's. We assume $\mu$ to be stationary and ergodic with respect to the shift $T: T x=\left\{x_{n+1}\right\}_{-\infty}^{\infty}$. Denote by $C$ a "chain" $C=\left(x_{1}, x_{2}, \ldots, x_{n}\right)$, of length $n$. The entropy $H$ of $\mu$ is defined as

where

$$
H=\lim _{n \rightarrow \infty} \frac{H_{n}}{n}=\sup _{n} \frac{H_{n}}{n}
$$

$$
H_{n}=-\sum c_{n} \mu\left(C_{n}\right) \log \mu\left(C_{n}\right) .
$$

McMillan's basic theorem now describes how many $C_{n}$ that actually occur.

Theorem. Given $\varepsilon>0$, there is $n_{0}(\varepsilon)$ so that for $n>n_{0}(\varepsilon)$, we can find distinct chains $C_{n 1}, C_{n 2}, \ldots, C_{n m}$ of length $n$ with $m<e^{n(H+\varepsilon)}$ so that

$$
\sum_{j=1}^{m} \mu\left(C_{n j}\right)>1-\varepsilon
$$

It is clear that this is a description of the support of $\mu$. Our scheme is therefore to relate harmonic measure to a stationary $\mu$ for which we can actually compute the entropy. We also obtain a connection to the geometry of the situation. Since the sup- 
port of our harmonic measure in the sense of distributions is the entire set we have to make a more careful definition.

Let $w$ be compactly supported positive measure. We say that the support $S(\omega)$ of $\omega$ has dimension $\alpha$ if for every $\beta>\alpha$ and every $\varepsilon>0$ we can find discs $D_{v}$ of radii $r_{v}$ so that

$$
\begin{gathered}
\sum r_{v}^{\beta}<\varepsilon, \\
\omega\left(\boldsymbol{R}^{2} \backslash \cup D_{v}\right)<\varepsilon,
\end{gathered}
$$

while this fails for every $\beta<\alpha$.

2. Let us begin by presenting a simple proof of Øksendal's theorem.

$\varnothing \mathrm{ksendal}$ 's theorem. Let $E$ be a compact set in the plane with connected complement. Then $S\left(\omega_{E}\right)$ has two-dimensional Lebesgue measure zero.

Lemma. Suppose $E \subset\{z|| z \mid<1\}$ and assume $m_{2} E \geqq \pi-\delta \pi, \delta>0$. Let $E_{0}=$ $E \cap\{z|1 / 2 \leqq| z \mid \leqq 1\}$. Then there is a universal constant $C$ so that

$$
\omega\left(z ; E_{0}\right) \geqq 1-C \delta \quad \text { on } \quad|z|=\frac{3}{4} .
$$

Proof. Choose a radius $r_{1}, 7 / 8<r_{1}<1$, so that

$$
m_{1}\left\{\theta \mid r_{1} e^{i \theta} \in E\right\} \geqq 2 \pi-\delta / 20
$$

and $r_{2}$ similarly in $1 / 2<r_{2}<5 / 8$. Let $G(z ; \zeta)$ be the Green's function for $R: r_{2}<$ $|z|<r_{1}$, and define in $R$

$$
u(z)=\frac{1}{2 \pi} \int_{\substack{\partial R \\ \zeta \notin E}} \frac{\partial G(z ; \zeta)}{\partial n_{\zeta}}|d \zeta| .
$$

By the maximum principle $\omega\left(z ; E_{0}\right)+u(z) \geqq 1$ in $R$. Writing down the explicit form of $G$ and using (1), we see that

so that

$$
u(z) \leqq \text { (Const.) } \delta \text { on }|z|=3 / 4
$$

$$
\omega\left(z ; E_{0}\right) \geqq 1-C \delta \text { on }|z|=3 / 4 .
$$

For the proof of the theorem we may of course assume that $m_{2} E>0$. Let $\omega$ denote harmonic measure for the complement of $E$, so that $\omega(z ; D), D \subset E$, is harmonic outside $E$ and $=1$ on $D,=0$ on $E \backslash D$. We shall prove that if $z_{0}$ is a point of density of $E$, then the Radon-Nikodym derivative of $\omega$ vanishes at $z_{0}$.

We take $z_{0}=0$. Let $B_{\varepsilon}=\{v|| v \mid<\varepsilon\}$ and define

and

$$
\varphi(\varepsilon)=\omega\left(B_{\varepsilon}\right)
$$

$$
\delta(\varepsilon)=1-\frac{m_{2}\left(E \cap B_{\varepsilon}\right)}{\pi \varepsilon^{2}} .
$$


By the lemma $\omega\left(z ; E \cap B_{\varepsilon / 2}\right) \leqq C \delta(\varepsilon)$ on $|z|=3 \varepsilon / 4$ so that for $|z|>\varepsilon$

$$
\omega\left(z ; E \cap B_{\varepsilon / 2}\right) \leqq \frac{C \delta(\varepsilon)}{1-C \delta(\varepsilon)} \omega\left(z ; E \cap B_{\varepsilon}\right) .
$$

This yields

$$
\varphi\left(\frac{\varepsilon}{2}\right) \leqq 2 \delta(\varepsilon) \varphi(\varepsilon)<\frac{1}{5} \varphi(\varepsilon)
$$

if $\delta(\varepsilon)<1 / 10$. At a point of density this holds for $\varepsilon<\varepsilon_{0}$ and hence

so that

$$
\varphi\left(\varepsilon_{0} 2^{-n}\right)<5^{-n}
$$

$$
\lim \frac{\varphi(\varepsilon)}{\varepsilon^{2}}=0
$$

3. Let $\boldsymbol{P}$ be a fixed simple polygon with $N$ sides and with the following relation to a basic directed line segment $S_{0}$ of length 1 .

1. The endpoints of $\boldsymbol{P}$ and $S_{0}$ coincide.

2. The first and the last side of $\boldsymbol{P}$ fall on $S_{0}$.

3. The sides $2, \ldots, N-1$ of $\boldsymbol{P}$ bound with $S_{0}$ a simply-connected polygon with negative orientation.

4. If the sides of $\boldsymbol{P}$ have lengths $p(1), p(2), \ldots, p(N)$ then $p(1), p(N)<1 / 2$ and $p(2), \ldots, p(N-1)<1$.

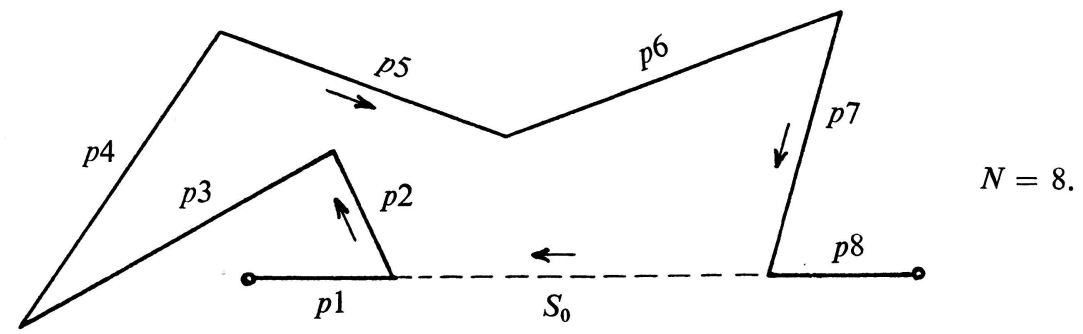

If $S$ is a directed line segment we denote by $P(S)$ the polygon similar to $P$ with base $S$ instead of $S_{0}$.

We start from any fixed simple polygon, say the unilateral triangle $\Delta_{0}$ bounding $\Omega_{0}$ and containing the origin. For each side $S$ of $\Delta_{0}$ we construct $\Sigma(S)$. We obtain a new polygon $\Delta_{1}$ with $3 N$ sides. These may intersect but because of our assumption 3 , $\Delta_{1}$ bounds a simply-connected domain $\Omega_{1} \supset \Omega$ on a Riemann surface. We continue the construction and obtain at the $n$-th stage a polygon $\Delta_{n}$ with $3 N^{n}$ sides bounding a domain $\Omega_{n}$. We note that by assumption 4 the $\Delta_{n}$ are all located in a fixed bounded domain and the sides of $\Delta_{n}$ tend to zero exponentially. $\Omega_{n}$ tends to a domain $\Omega$.

The or em. The support of harmonic measure inside $\Omega$ has dimension $\leqq 1$ on $\partial \Omega$. 
The boundary sides of $\Delta_{n}$ have a natural representation by a sequence $\left(x_{0}, x_{1}, \ldots\right.$, $x_{n}$ ) where $x_{0}=1,2,3$ and $x_{i}=1,2, \ldots, N, i \geqq 1$, indicates on which side the construction was made at the $i$-th stage. We are going to disregard the special role of $i=0$ in the notation. Each domain $\Omega_{n}$ has harmonic measure $\omega^{n}$ and $\omega^{n} \rightarrow \omega$ (weak-*) which is harmonic measure for the limit domain $\Omega$. The value of $\omega$ for a boundary sequence $C=x_{0}, x_{1}, \ldots, x_{n}$ is denoted $\omega(z ; C)$ and for $z=0$ we write simply $\omega(C)$.

To obtain a stationary measure we now introduce the space $X^{*}$ of doubly infinite sequences $X^{*}=\left\{x_{n}\right\}_{-\infty}^{\infty}$. The measure $\mu$ is defined in this space $X^{*}$ as follows:

Let $C$ be a chain in some arbitrary position in $X^{*}$. We extend $C$ to the left by a sequence $A$ of length $|A|=n$ obtaining after translation a new chain of length= $=n+$ length $(C)$ at position $\left(x_{0}, x_{1}, \ldots, x_{n+|C|-1}\right)$. We shall presently show that the following limit exists and gives a definition of a probability measure $\mu$ :

$$
\mu(C)=\lim _{n \rightarrow \infty} \sum_{A} \omega(A, C) .
$$

The definition is clearly translation invariant. Before proceeding let us drop the *-notation. All sequences will be doubly infinite but $\omega$ is naturally only used for sequences $\left(x_{0}, x_{1}, \ldots, x_{n}\right)$.

The existence and other basic properties of $\mu$ now follow from the following

Lemma 1.

$$
\frac{\omega(A, C, B)}{\omega(A, C)} \frac{\omega(C)}{\omega(C, B)} \rightarrow 1
$$

uniformly in $A$ and $B$ as $|C| \rightarrow \infty$.

Proof. Let us first disentangle the notation. For a chain $D, \omega(D)$ denotes the value at $z=0$ of the harmonic measure $\omega(z ; D)$. In the lemma, $B$ denotes a sequence of choices of sides and the lemma means that the relative harmonic measure

$$
\frac{\omega(z ; C, B)}{\omega(z ; C)} \quad(z=0)
$$

is not influenced by earlier conditionings (by $A$ ).

To prove the lemma let us map the situation to the unit disc. The length-area proof of the Carathéodory theorem applies and we conclude that the mapping is bicontinuous. Let $C$ have length $n, C=\left(c_{1}, \ldots, c_{n}\right)$. Choose the mapping so that the midpoint of the segment $S=\left(c_{1}, \ldots, c_{n / 2}\right)$ is mapped to $\zeta=0$. $C$ defines an arc $\gamma$ on $|\zeta|=1$ and $(C, B)$ an arc $\beta \subset \gamma$. Then $u(\zeta ; \gamma)$ and $u(\zeta ; \beta)$ are the corresponding harmonic measures

$$
u(\zeta ; \gamma)=\frac{1}{2 \pi} \int_{\gamma} \frac{1-|\zeta|^{2}}{\left|\zeta-e^{i t}\right|^{2}} d t .
$$

It is clear from the geometric construction of $\Sigma(S)$ that the preimage $\sigma$ of $S$ in $|\zeta|<1$ satisfies, since the mapping is continuous,

$$
|\gamma|=(\text { length } \gamma)=o(\operatorname{dist}(\sigma, \gamma)), \quad n \rightarrow \infty,
$$


uniformly in all choices of $C$ and $B$. This implies that

$$
\lim _{n=\infty} \frac{u(\zeta ; \beta)}{u(\zeta ; \gamma)}=\lim _{n=\infty} \frac{|\beta|}{|\gamma|}=q
$$

uniformly for $\zeta \in \sigma$. Hence

$$
(q-\varepsilon) \omega(z ; C) \leqq \omega(z ; C, B) \leqq(q+\varepsilon) \omega(z ; C)
$$

"inside" the cross-section $S$ and in particular for $z=0$. If we add to $C$ the sequence $A$ but otherwize do the same construction we obtain the same limit $q$ and putting $z=0$ we get the lemma.

Let us now prove that the limit (1) exists. We write $A=A^{\prime}, A_{0}$ and keep $A_{0}$ fixed and long. Then

$$
\omega(A, C)=\omega\left(A^{\prime}, A_{0}, C\right)=\frac{\omega\left(A_{0}, C\right)}{\omega\left(A_{0}\right)} \omega\left(A^{\prime}, A_{0}\right)(1+\varepsilon)
$$

where $\varepsilon\left(A^{\prime}\right) \rightarrow 0$, as $\left|A_{0}\right| \rightarrow \infty$. Hence

$$
\sum_{A^{\prime}} \omega\left(A^{\prime}, A_{0}, C\right)=\omega\left(A_{0}, C\right)\left(1+\varepsilon^{\prime}\right)
$$

which proves the existence of $\mu$. Actually we have the stronger result

$$
\lim _{m \rightarrow \infty} \frac{\sum_{|A|=m} \omega(A, C)}{\mu(C)}=1
$$

uniformly in $C$.

Lemma 2. The measure $\mu$ is a probability measure with the following properties:

(a) $\mu$ is invariant under $T$

$$
\mu(T C)=\mu(C),
$$

(b) $\mu$ is equivalent to $\omega$, i.e.

Const. $\omega(C) \leqq \mu(C) \leqq$ Const. $\omega(C)$,

(c) $\mu$ is ergodic for $T$.

Proof. We have already observed that the definition of $\mu$ makes sense. We also have $\sum_{|C|=k} \mu(C)=1$ since this holds for $\omega$ and $\mu$ extends to a probability measure on $X^{*}$. Because of (2) $\mu$ is equivalent to a fixed finite sum $\sum_{A} \omega(A, C)$ and this is clearly equivalent to $\omega(C)$.

To prove (c) let us consider a cylinder set defined by $C$ and $C^{\prime}$ at distance $m$ from each other. Let us consider $D=A C B C^{\prime},|B|=m,|A|=n$. On cross-sections $\beta$ in $B$

$$
\frac{\omega\left(z ; A C B C^{\prime \prime}\right)}{\omega\left(z ; A C B C^{\prime}\right)} \leqq \text { Const. } \frac{\mu\left(C^{\prime \prime}\right)}{\mu\left(C^{\prime}\right)}
$$


with a uniform constant. It now follows that

$$
\sum_{|B|=m} \mu\left(C B C^{\prime}\right) \geqq \text { Const. } \mu(C) \mu\left(C^{\prime}\right)
$$

uniformly in $m$. This weak form of mixing implies ergodicity. This is certainly wellknown. For convenience, a sketch of a proof is included.

Suppose $E$ is an invariant set with $0<\mu(E)<1$ and let $F$ be the complement of $E$. Let $A$ and $B$ be cylinder approximations to $E$ and $F$ and translate $B$ by $N$ so that $A$ and $B$ have disjoint positions. Then

but also

$$
\mu(A \cap B) \leqq \mu\left(E \cap T^{-N} F\right)+\varepsilon \leqq \mu(E \cap F)+\varepsilon=\varepsilon
$$

$$
\mu(A \cap B) \geqq \text { Const. } \mu(A) \mu(B) \geqq \text { Const. }(\mu(E)-\varepsilon)(\mu(F)-\varepsilon) .
$$

Hence $\mu(E)$ or $\mu(F)=0$.

Let us now compute the entropy of $\mu$. We fix some large $m$ so that

for all $C$. Then

$$
1-\varepsilon \leqq \frac{\sum_{|A|=m} \omega(A, C)}{\mu(C)} \leqq 1+\varepsilon
$$

$$
\begin{aligned}
& H_{n}=-\sum_{|C|=n} \mu(C) \log \mu(C) \\
& \leqq(1+\varepsilon) \sum_{|C|=n}\left(-\sum_{|A|=m} \omega(A, C)\right) \log \left(\sum_{|A|=m} \omega(A, C)\right)+(1+\varepsilon) \log (1+\varepsilon) \\
& \leqq(1+\varepsilon) \sum_{|C|=n+m}(-\omega(C) \log \omega(C))+(1+\varepsilon) \log (1+\varepsilon)
\end{aligned}
$$

since $f(x)=-x \log x$ satisfies $f\left(x_{1}+x_{2}+\ldots+x_{n}\right) \leqq \sum_{v=1}^{n} f\left(x_{v}\right)$. Hence

$$
H=\lim _{n=\infty} \frac{H_{n}}{n} \leqq \lim -\frac{1}{n} \sum_{\omega_{n}} \omega_{n} \log \omega_{n}
$$

where $\omega_{n}=\omega(C)$ denote the harmonic measure of the boundary intervals $\sigma_{n}$ at the $n$-th stage of the construction. Formally

$$
\omega_{n}=\frac{1}{2 \pi} \int_{\sigma_{n}} \frac{\partial g}{\partial n} d s
$$

where $g$ is the Green's furction of the limit domain with pole at 0 . Let us stop the construction at same stage $k>n$. Then the formula is valid and if we make estimates independent of $k$, these estimates will hold for the limit domain.

Let us consider

$$
E(\sigma)=\frac{1}{2 \pi} \int_{\sigma} \frac{\partial g}{\partial n} \log \frac{\partial g}{\partial n} d s=\frac{1}{2 \pi} \int_{\sigma}\left(\frac{\partial g}{\partial n} \delta\right)\left(\log \frac{\partial g}{\partial n} \delta\right) \frac{d s}{\delta}-\omega_{n} \log \delta
$$

where $\delta$ is the length of the arc $\sigma$.

The first integral $I$ is scale invariant and we may assume that our basic interval is $(0,1)$ on the real axis. Let $F(\zeta)=Z$ be the conformal mapping from $\eta>0$ to the 
domain with $F(i)=0$ and $F(0) \in \sigma$. Then

$$
I=\frac{1}{\pi} \int_{F^{-1}(\sigma)}-\log \left(\left|F^{\prime}(x)\right|\left(1+x^{2}\right)\right) \frac{d x}{1+x^{2}} .
$$

Here $F^{-1}(\sigma)=\tau$ is an interval of length $l(\leqq 1$, say) and

$$
\begin{gathered}
\omega=\omega_{n}=\frac{1}{\pi} \int_{\tau} \frac{d x}{1+x^{2}} \sim \frac{l}{\pi}, \\
I=G(\omega)-\frac{1}{\pi} \int_{\tau} \frac{\log \left|F^{\prime}(x)\right| d x}{1+x^{2}}=(G(\xi)=F(l \xi)) \\
=O(\omega)-l \frac{1}{\pi} \int_{\tau_{0}} \frac{\log \left|G^{\prime}(\xi)\right| d \xi}{1+l^{2} \xi^{2}}+\omega \log \omega .
\end{gathered}
$$

Since $F$ is bounded $\left|G^{\prime}(i)\right| \leqq$ Const. Here $\tau_{0}$ is an interval of length 1 containing $\xi=0$. We shall prove that integrals of this type are bounded.

Lemma 3. Let $G(\zeta)$ be univalent in $\eta>0$ and smooth up to $\eta=0$ and assume $\left|G^{\prime}(i)\right| \leqq 1$. Let $\psi(\zeta) \in C^{2}$ in $\eta \geqq 0$. Then there is universal constant $C$ so that

$$
\int_{-1}^{1} \psi(\xi) \log \left|G^{\prime}(\xi)\right| d \xi \leqq C\|\psi\|_{C^{2}} .
$$

Proof. We may of course assume $\psi(\zeta)=0,|\zeta|>2$. Set

and

$$
U(\xi, \eta)=\frac{1}{\pi}\left(\operatorname{arctg} \frac{\xi-1}{\eta}-\operatorname{arctg} \frac{\xi+1}{\eta}\right)
$$

$$
u(\xi, \eta)=\eta \psi(\zeta) U(\xi, \eta)
$$

By Green's formula

$$
\int_{-1}^{+1} \psi(\xi) \log \left|G^{\prime}(\xi)\right| d \xi=\iint_{\eta>0} \log \left|G^{\prime}(\zeta)\right| \Delta u(\zeta) d \zeta d \eta
$$

To obtain the desired result we now only have to use the estimates in $|\zeta|<2$ :

$$
\begin{gathered}
|\log | G^{\prime}(\zeta) \mid \leqq \text { Const. } \log \frac{3}{\eta}, \\
|\Delta u| \leqq \text { Const. } \frac{1}{\left|\zeta^{2}-1\right|}, \\
\iint_{|\zeta|<2} \log \frac{3}{\eta} \frac{d \xi d \eta}{\left|\zeta^{2}-1\right|}<\infty .
\end{gathered}
$$

The proof of the lemma is complete.

The result of the computation is that, from (2),

$$
E(\sigma)=\omega \log \omega-\omega \log \delta+O(\omega)
$$


for all $\sigma=\sigma_{n}$ in the $n$-th generation. We also observe that

$$
\sum_{\sigma_{n}} E\left(\sigma_{n}\right)=\frac{1}{2 \pi} \int \frac{\partial g}{\partial n} \log \left|G^{\prime}\right| d s=\log \left|G^{\prime}(0)\right|
$$

where $G$ is the conformal mapping to $|\zeta|<1$. Hence

and

$$
H_{n} \leqq-\sum_{\omega_{n}}\left(\log \delta_{n}\right) \omega_{n}+O(1)
$$

$$
H \leqq-\varlimsup \frac{1}{n} \sum_{\omega_{n}}\left(\log \delta_{n}\right) \omega_{n} .
$$

We now observe that

$$
\delta_{n}=\prod_{i=1}^{n} p\left(x_{i}\right) .
$$

Since $\mu$ and $\omega$ are equivalent measures we can use the individual ergodic theorem for $\mu$ to conclude that

$$
e^{-n(K-\varepsilon)}<\delta_{n}<e^{-n(K+\varepsilon)}
$$

for all $\delta_{n}$ except a set of small harmonic measure. It follows from (3) that $K \geqq H$. On the other hand by the McMillan theorem only $e^{(H+\varepsilon) n}$ intervals are needed to cover essentially all harmonic measure. We conclude that the $(1+2 \varepsilon)$-dimensional Hausdorff measure of the support is zero.

4. Let us now consider the case of the exterior of a two-dimensional Cantor set. In this case the $n$-th stage consists of $4^{n}$ squares of sides $a^{n}, a<1 / 2$ and we want to prove the following.

Theorem. For any two-dimensional Cantor set the support of harmonic measure has dimensicn $<1$.

The previous proof applies with two rather essential changes. Lemma 1 cannot be proved using conformal mapping and the computation of the entropy is different since $\log |\nabla g|$ has singularities.

A substitute for Lemma 1 is given in the following

Lemma 5. Let $D$ be a domain in the complex plane. $A$ is a conformal annulus, $A=B \backslash C, B, C$ conformal disks, $E \subset \partial D$ is such that $E \subset C_{1} \subset B_{1} \subset C_{2} \subset B_{2} \subset \ldots$ $\ldots \subset C_{n} \subset B_{n}$ where the annuli $A_{v}=B_{v} \backslash C_{v}$ are similar to $A$ and $A_{v} \subset D$. Let $u$ and $v$ be non-negative harmonic functions in $D$ so that $u$ and $v=0$ on $\partial D \backslash E$. Then there is a constant $C_{0}$, independent of $u$ and $v$ and another constant $C$, depending on $u$ and $v$ but not on $n$ so that

$$
\left|C \frac{u(z)}{v(z)}-1\right| \leqq \frac{C_{0}}{n^{2}} \text { in } A_{n}
$$

and hence in the part of $D$ exterior to $B_{n}$. 
Proof. In each annulus $A_{v}$ pick a smooth curve $\gamma_{v}$ so that all the $\gamma_{v}$ are similar. We define

$$
M_{v}=\sup _{\gamma_{v}} \frac{u(z)}{v(z)}, \quad m_{v}=\inf _{\gamma_{v}} \frac{u(z)}{v(z)}, \quad Q_{v}=\frac{M_{v}}{m_{v}} .
$$

From the assumption it follows that $M_{v} \searrow$ and $m_{v} \nearrow$. We shall study the sequence $Q_{v}$. Consider $v<n$ fixed. By multiplying $u$ and $v$ by constants we may assume that $M_{v}=1$ and $\operatorname{Max}_{\gamma_{v}} u(z)=1$. We may also assume that $\gamma_{v}$ has length 1. By Harnack's inequality $u(z) \leqq c_{1}$ on $\gamma_{v}$. It also follows that $\left|\nabla^{2} u\right|,\left|\nabla^{2} v\right| \leqq c_{2}$ on $\gamma_{v}$. Let $z_{0}$ be a point on $\gamma_{v}$ where

We conclude that

$$
Q_{v} u\left(z_{0}\right)=v\left(z_{0}\right)
$$

$$
v \geqq\left(Q_{v}-1\right) c_{3}+u
$$

on an interval $I$ on $\gamma_{v}$ of length $\geqq\left(Q_{v}-1\right)^{1 / 2} C_{4}$. Now let $G_{v}$ be the Green's function for $D_{v}=D \backslash\left(\right.$ inside of $\left.\gamma_{v}\right)$. For $z \in D_{v}$

$$
\begin{gathered}
u(z)=\frac{1}{2 \pi} \int_{\gamma_{v}} \frac{\partial G_{v}}{\partial n}(z ; \zeta) u(\zeta) d s_{\zeta} \\
\leqq \frac{1}{2 \pi} \int_{\gamma_{v}} \frac{\partial G_{v}}{\partial n}(z ; \zeta) v(\zeta) d s_{\zeta}-\frac{1}{2 \pi} \int_{I} \frac{\partial G_{v}}{\partial n}(z ; \zeta) C_{3}\left(Q_{v}-1\right) d s_{\zeta}
\end{gathered}
$$

For $z \in \gamma_{v+1}, \frac{\partial G_{v}}{\partial n}$ satisfies $\frac{\sup _{\gamma_{v}} \partial G_{v} / \partial n}{\inf _{\gamma_{v}} \partial G_{v} / \partial n} \leqq C_{5}$. We conclude that on $\gamma_{v+1}$

Hence

$$
u(z) \leqq v(z)\left(1-C_{6}\left(Q_{v}-1\right)^{3 / 2}\right)
$$

and so

$$
M_{v+1} \leqq M_{v}\left(1-C_{6}\left(Q_{v}-1\right)^{3 / 2}\right)
$$

$$
Q_{v+1}=\frac{M_{v+1}}{m_{v+1}} \leqq \frac{M_{v+1}}{m_{v}} \leqq Q_{v}\left(1-C_{6}\left(Q_{v}-1\right)^{3 / 2}\right)
$$

This iteration is easily estimated and we find

$$
0 \leqq Q_{n}-1 \leqq \frac{C_{0}}{n^{2}}
$$

and Lemma 5 is proved.

For the entropy we get the following formula

$$
\frac{1}{2 \pi} \int_{\Gamma_{n}} \frac{\partial g}{\partial n} \log |\nabla g| d s=\sum_{(n)} g\left(\zeta_{v}\right)+\text { Const. }
$$

where $\zeta_{v}$ are the critical points of $g$ and $(n)$ indicates that we should sum over those $\zeta_{v}$ which are inside the $n$-th approximation of the Cantor region. For the final inequality 
we get

$$
\frac{H_{n}}{n} \leqq(-\log a)-\frac{1}{n} \sum_{(n)} g\left(\zeta_{v}\right)
$$

The theorem therefore follows from the following.

Lemma 6. There is a constant $c>0$ so that

$$
\sum_{(n)} g\left(\zeta_{v}\right) \geqq c n
$$

Proof. (The idea of the proof is due to Peter Jones.)

Let us consider the $i$-th stage in the construction of the Cantor set and a square $Q_{i}$ of the corresponding generation. We shall prove that we associate to every $Q_{i}^{0}$ one $\zeta_{v}$ so that $v=v\left(Q_{i}^{0}\right)$,

$$
g\left(\zeta_{v}\right) \geqq \text { Const. } \omega\left(Q_{i}\right)
$$

and so that the same $\zeta_{v}$ is only used four times.

We consider the configuration of 4 squares $Q$, related to $Q_{i}^{0}$ :

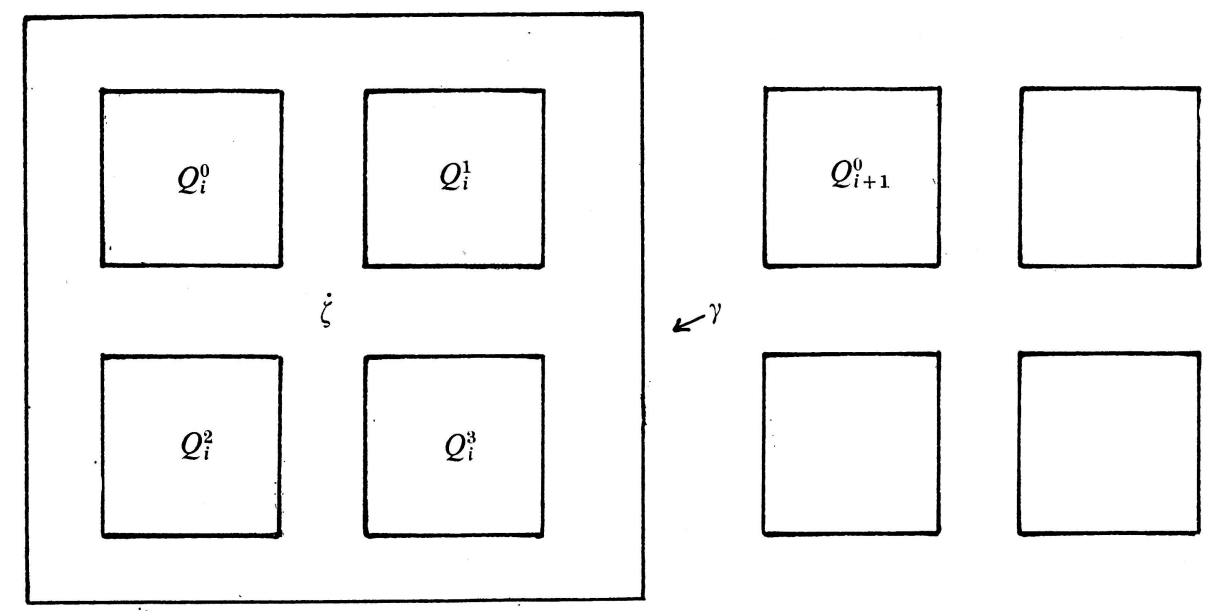

By Harnack's inequality $g(z ; \zeta)$ is comparable to harmonic measure for all four $Q_{i}^{k}, k=0,1,2,3$, on $\gamma$ around the $Q_{i}^{k}$ :s. Hence

$$
g(\infty ; \zeta)=g(\zeta ; \infty) \asymp \omega\left(Q_{i}^{k}\right), \quad k=0,1,2,3 .
$$

It now follows that there is also a critical point inside $\gamma$ which has the same property at a point where level lines of $g \asymp g(\zeta ; \infty)$ meet.

We now sum and find

$$
\sum_{Q_{i}} g\left(\zeta_{v}\left(Q_{i}\right)\right) \geqq c_{0}
$$

and summation over $i$ gives the lemma. 
Added in proof. N. G. Makarov has proved that the dimension of support of harmonic measure is $\leqq 1$ for all simply-connected domains.

\section{References}

[1] Billingsley, P.: Ergodic theory and information. - John Wiley \& Sons, Inc., New YorkLondon-Sydney, 1965.

[2] Kaufman, R., and J.-M. Wu: On the snowflake domain. - Ark. Mat. (to appear).

[3] Manning, A.: The dimension of the maximal measure for a polynomial map. - Preprint, Warwick, 1983.

[4] ØKSENDAL, B.: Brownian motion and sets of harmonic measure zero. - Pacific J. Math. 95, 1981, 179-192.

Institut Mittag-Leffler

Auravägen 17

S-18262 Djursholm

Sweden

Received 5 April 1984 